\title{
Transsylvian and trans-Heschl's gyrus approach for a left posterior insular lesion and functional analyses of the left Heschl's gyrus: illustrative case
}

\author{
Shunsuke Nakae, MD, MSc, PhD, ${ }^{1}$ Masanobu Kumon, MD, ${ }^{1}$ Daijiro Kojima, MD, ${ }^{1}$ Saeko Higashiguchi, MD, ${ }^{1}$ Shigeo Ohba, MD, PhD, \\ Naohide Kuriyama, MD, PhD, ${ }^{2}$ Yuriko Sato, SLHT, MS, ${ }^{3}$ Yoko Inamoto, SLHT, PhD, ${ }^{3}$ Masahiko Mukaino, MD, $\mathrm{PhD},{ }^{3}$ and \\ Yuichi Hirose, MD, PhD ${ }^{1}$
}

Departments of ${ }^{1}$ Neurosurgery and ${ }^{2}$ Anesthesiology, Fujita Health University, Toyoake, Aichi, Japan; and ${ }^{3}$ Rehabilitation Complex, Fujita Health University, Toyoake, Aichi, Japan

\begin{abstract}
BACKGROUND A common surgical approach for dominant insular lesions is to make a surgical corridor in asymptomatic cortices based on functional mapping. However, the surgical approach is difficult for posterior insular lesions in a dominant hemisphere because the posterior parts of the perisylvian cortices usually have verbal functions.

OBSERVATIONS We present the case of a 40-year-old male whose magnetic resonance images revealed the presence of contrast-enhancing lesions in the left posterior insula. Our surgical approach was to split the sylvian fissure as widely as possible, and partially resect Heschl's gyrus if the cortical mapping was negative for language tests. Because Heschl's gyrus did not have verbal functions, the gyrus was used as a surgical corridor. It was wide enough for the removal of the lesion; however, because intraoperative pathological diagnosis eliminated the possibility of brain tumors, further resection was discontinued. The tissues were histologically diagnosed as tuberculomas. Antituberculosis drugs were administered, and the residual lesions finally disappeared. According to the neurophysiological tests, the patient showed temporary impairment of auditory detection, but the low scores of these tests improved.
\end{abstract}

LESSONS The transsylvian and trans-Heschl's gyrus approach can be a novel surgical option for excising dominant posterior insular lesions.

https://thejns.org/doi/abs/10.3171/CASE21622

KEYWORDS awake craniotomy; brain mapping; posterior insular lesions; surgical approach; Heschl's gyrus; neurophysiological tests

Resection of an insular lesion in a dominant hemisphere is challenging. Surgical approaches to the insula, mostly in cases of insular gliomas, have been discussed in many articles. ${ }^{1-4}$ The dominant insular lesion is usually approached by creating transcortical windows in the cortices above and below the sylvian fissure after functional mapping in an awake condition, although small differences exist in the surgical procedures, for example, with or without sylvian fissure dissections. Because the posterior parts of the dominant perisylvian cortices usually have verbal functions, it is difficult to approach the posterior insular lesion by removing the operculum to create surgical windows. Moreover, the posterior portion of the insula is deeper than the anterior portion, and the superficial sylvian vein is frequently bifurcated in the posterior part of the sylvian fissure, which complicates dissection. ${ }^{5}$ To the best of our knowledge, few articles have discussed the approaches to insular lesions in cases in which they are located in the posterior superior temporal gyrus (STG), which has verbal functions.

Heschl's gyrus, which corresponds to Broadman's areas 41 and 42 , is the longest and the most anterior gyrus of the transverse temporal gyri and is located adjacent to the planum temporale. ${ }^{6}$ Heschl's gyrus is also known as the primary auditory area, and a recent study has indicated that it functions as the auditory-language hub. ${ }^{7}$ However, other functions of Heschl's gyrus are yet to be elucidated. In this article, we have reported a safe approach to a lesion located in the posterior insula through intraoperative brain mapping of the perisylvian cortices, including Heschl's gyrus, and 

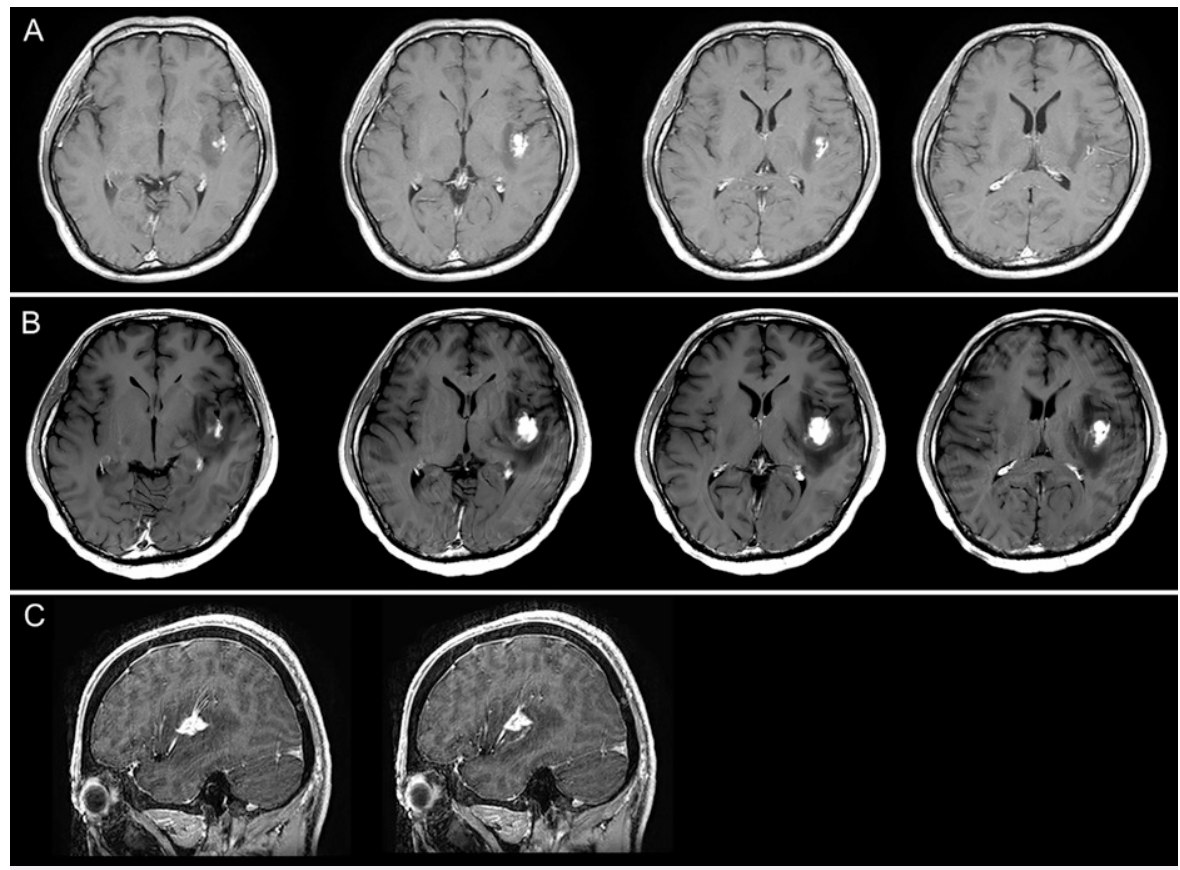

FIG. 1. A: Contrast-enhanced magnetic resonance (MR) images of the posterior insular lesion at first detection. Axial (B) and sagittal (C) MR images of the insular lesion 2 weeks after the first detection.

the removal of a safe region of the gyrus to create a surgical corridor. Moreover, based on the results of brain mapping and neurophysiological tests, the detailed functions of the dominant Heschl's gyrus have been discussed in this report.

\section{Illustrative Case}

A 40-year-old right-handed male without a history of other diseases visited a hospital because of complaint of a headache. Magnetic resonance imaging (MRI) results revealed a contrast-enhancing mass in the left posterior insula with accompanying edema (Fig. 1A). He was admitted to our institution 2 weeks after the detection of the lesion, and the MRI was repeated. The finding revealed that the lesion and perifocal edema in the posterior region of the left insula were enlarged when compared with the previous images (Fig. 1B and C). Because high-grade glioma was suspected, we recommended to the patient and his family that resection by awake craniotomy be performed at the earliest, to which they agreed. The contrast-enhancing lesion was located in the posterior portion of long gyri of the left insula, and the edema in the temporal operculum posed difficulties in exposing the insular cortex by fully splitting the sylvian fissure (Fig. 2A). Because the images in the MRI fiber-tracking suggested that a part of the left Heschl's gyrus might be resected (Fig. 2B), our surgical plan was to split the sylvian fissure as widely as possible, expose the cortex of Heschl's gyrus and the insula, and remove a part of Heschl's gyrus to create a surgical corridor if the brain mapping results were negative for language tests. Neuropsychological tests were performed as part of
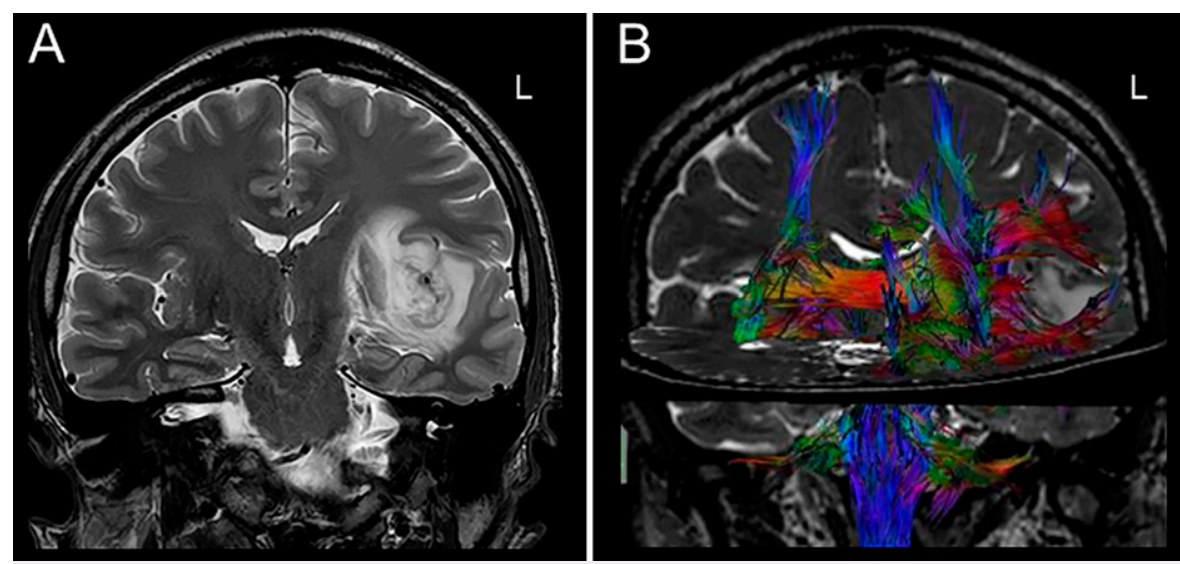

FIG. 2. A: According to a coronal T2-weighted image of the insular lesion, sylvian fissure dissection seemed to be challenging due to extensive perifocal edema. B: A fiber-tracking image suggested that Heschl's gyrus could be a surgical window. $L=$ left side in both images. 
the preoperative evaluation of brain functions, the results of which are summarized in Table 1.

Transcranial motor evoked potentials and electrocorticography were used to detect hemiparesis and ictal states during the operation, respectively. The surgical procedures were as follows. After frontotemporal craniotomy under general anesthesia, an antiepileptic drug was intravenously administered. The perisylvian cortices were exposed (Fig. 3A), the distal sylvian fissure was split as widely as possible without cutting the superficial sylvian bridging veins, and a part of the insular cortices was exposed (Fig. 3B-D). Thereafter, a part of the insular cortices that was hyperintense on T2-weighted imaging was sampled for the intraoperative histological diagnosis, which revealed that it was almost normal brain. Subsequently, the patient was woken for intraoperative brain mapping. The tasks for the verbal mapping included naming, reading,

TABLE 1. Preoperative and postoperative evaluations of neurophysiological tests

\begin{tabular}{|c|c|c|c|}
\hline Examinations & Preoperative Score & Postoperative Score (2 weeks) & Postoperative Score (4 months) \\
\hline MMSE & $30 / 30$ & $29 / 30$ & $30 / 30$ \\
\hline FAB & $17 / 18$ & $15 / 18$ & ND \\
\hline \multicolumn{4}{|l|}{ CAT } \\
\hline \multicolumn{4}{|l|}{ 1. Span } \\
\hline \multirow[t]{2}{*}{ Digit span } & Forward: 7-digit & Forward: 5-digit & Forward: 7-digit \\
\hline & Backward: 7-digit & Backward: 3-digit & Backward: 8-digit \\
\hline \multirow[t]{2}{*}{ Tapping span } & Forward: 9-digit & Forward: 6-digit & Forward: 9-digit \\
\hline & Backward: 9-digit & Backward: 9-digit & Backward: 9-digit \\
\hline \multicolumn{4}{|c|}{ 2. Cancellation and detection test } \\
\hline Visual cancellation task & $100 \% *$ & $100 \% *$ & $100 \% *$ \\
\hline \multirow[t]{2}{*}{ Auditory detection task } & $98 \% *$ & $58 \% *$ & $100 \% *$ \\
\hline & $100 \% \dagger$ & $53 \% \dagger$ & $100 \% \dagger$ \\
\hline 3. SDMT (achievement rate) & $70 \%$ & $68 \%$ & $76 \%$ \\
\hline \multicolumn{4}{|l|}{ 4. Memory updating test } \\
\hline 3-span & $68 \% *$ & $93 \% *$ & $94 \% *$ \\
\hline 4-span & $68 \% *$ & $37 \% *$ & $69 \% *$ \\
\hline \multicolumn{4}{|l|}{ 5. PASAT } \\
\hline 2-second condition & $80 \% *$ & $38 \% *$ & $92 \% *$ \\
\hline 1-second condition & $68 \% *$ & $23 \% *$ & $73 \% *$ \\
\hline 6. Position stroop test & $100 \% *$ & $100 \% *$ & $100 \% *$ \\
\hline \multicolumn{4}{|l|}{ RBMT } \\
\hline Standard profile & $23 / 24$ & $21 / 24$ & $22 / 24$ \\
\hline Screening & $11 / 12$ & $10 / 12$ & $10 / 12$ \\
\hline \multicolumn{4}{|l|}{ ROCFT } \\
\hline Copying & $36 / 36$ & $36 / 36$ & ND \\
\hline Immediate recall & $36 / 36$ & $36 / 36$ & ND \\
\hline Delayed recall & $36 / 36$ & $36 / 36$ & ND \\
\hline MoCA-J & $28 / 30$ & $29 / 30$ & ND \\
\hline \multicolumn{4}{|l|}{ SALA (naming) } \\
\hline High familiarity & $47 / 48$ & $47 / 48$ & ND \\
\hline Low familiarity & $45 / 48$ & $45 / 48$ & ND \\
\hline 2-mora & $29 / 30$ & $30 / 30$ & ND \\
\hline 3-mora & $30 / 30$ & $30 / 30$ & ND \\
\hline 4-mora & $29 / 30$ & $30 / 30$ & ND \\
\hline WAB AQ & $89.7 / 90$ & $84.2 / 90$ & $89.6 / 90$ \\
\hline \multicolumn{4}{|l|}{ SLTA } \\
\hline Sentence repetition & $4 / 5$ & $3 / 5$ & $4 / 5$ \\
\hline
\end{tabular}

$\mathrm{AQ}=$ Aphasia Quotient; CAT = Clinical Assessment for Attention; FAB = frontal assessment battery; MoCA-J = Japanese version of Montreal Cognitive Assessment; MMSE = Mini Mental State Examination; ND = no data; PASAT = Paced Auditory Serial Addition Test; RBMT = The Rivermead Behavioural Memory Test; ROCFT = Ray-Osterrieth Complex Figure Test; SALA = Sophia Analysis of Language in Aphasia; SDMT = Symbol Digit Modalities Test; SLTA = Standard Language Test of Aphasia; WAB = Western Aphasia Battery (Japanese version).

* The percentages show the correct answer rates.

$\dagger$ The percentages show the hitting ratios. 

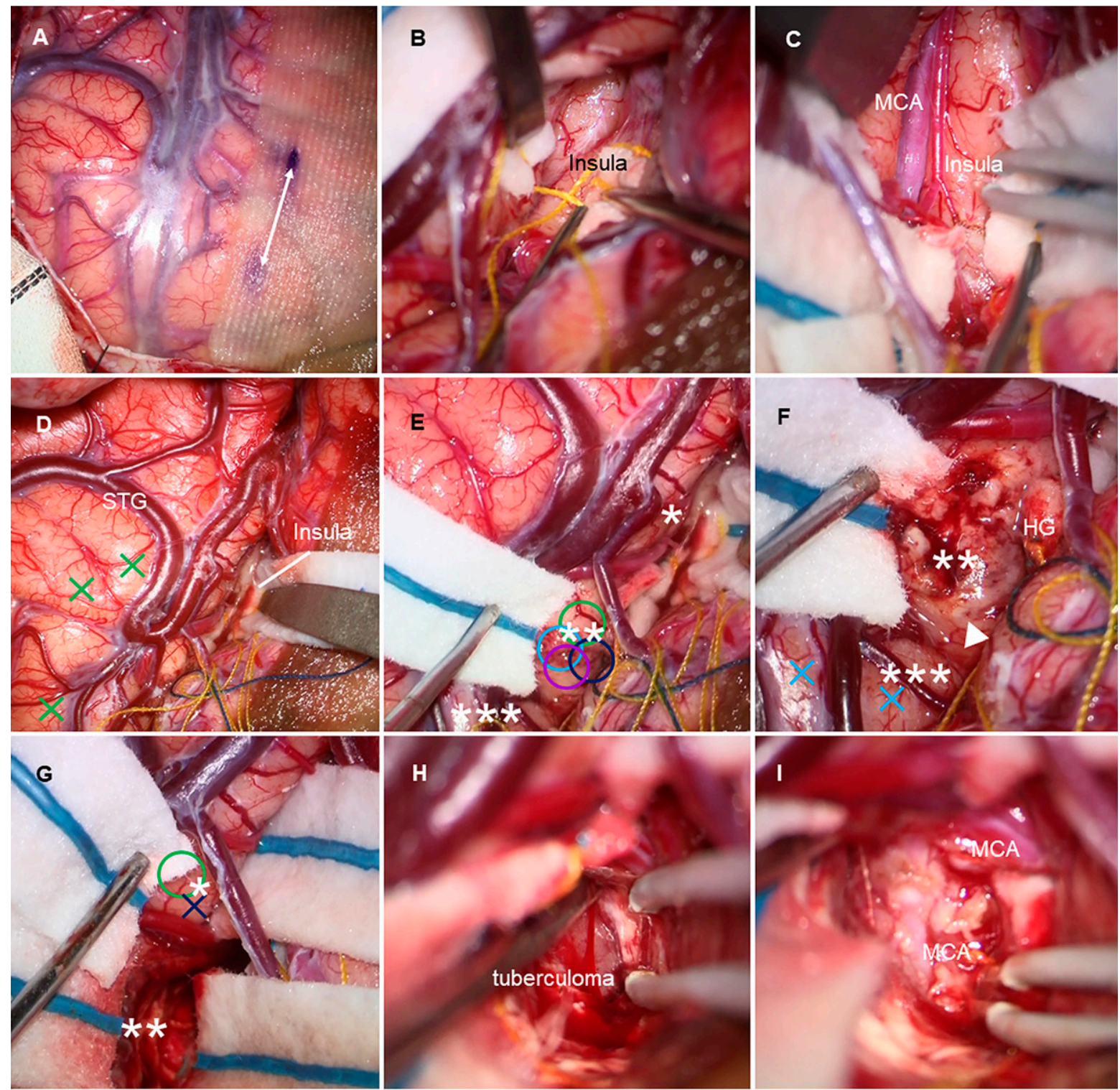

Asymptomatic areas against stimulation.

X Symptomatic areas against stimulation.

Each color indicates tasks for verbal functions as follows:

Green $=$ naming, sky blue $=$ repetition, navy $=$ auditory comprehension, purple $=$ reading.

FIG. 3. A-I: Intraoperative pictures. Two-headed arrow (A) indicates a putative location of the lesion according to a navigation system. The insular cortex was exposed after sylvian fissure dissection (B and $C$ ). The patient was then woken, and brain mapping was started. The results of the brain mapping is shown $(D-G)$. Each color indicates the tasks performed during the surgery: green = naming, sky blue $=$ repetition, navy $=$ auditory comprehension, and purple $=$ reading. According to the results of cortical mapping, each gyrus was considered as follows (E-G): * = STG, ** = Heschl's gyrus (HG), and ***= transverse temporal gyrus. The arrowhead (F) indicates the sulcus of Heschl. After the resection of Heschl's gyrus, the lesion (tuberculoma) was visualized $(\mathrm{H})$, and the MCA involved in the lesion was whitish (I). MCA = middle cerebral artery.

auditory comprehension, repetition, and free conversation. Direct electrical stimulation was performed, which started at $6 \mathrm{~mA}$ and reached a maximum of $10 \mathrm{~mA}$. Cortical mapping was initiated from the posterior STG, and the patient consequently exhibited paraphasia and word-finding difficulty during the stimulation of the posterior STG. Next, the cortex of Heschl's gyrus was exposed (Figs. $3 E$ and F), and a sulcus, supposedly the sulcus of Heschl, was detected (triangle in Fig. 3F). Cortical mapping was initiated at an anterior part of the cortex from the sulcus (area ** in Fig. 3E), which did not result in any symptoms during the stimulation. In contrast, during the stimulation of the posterior cortex of 
the sulcus (area $* * *$ in Fig. 3F), the patient could not successfully accomplish the repeating and reading tasks. Thus, we left this area intact, and corticectomy was performed throughout the cortex (area **). During corticectomy, the patient did not manifest any verbal symptoms. Although a part of the cortex of Heschl's gyrus was used as a surgical corridor, it was quite narrow for the removal of the lesion in the insula. Therefore, we stimulated the anterior cortex from the surgical window (area * in Fig. 3G) to enlarge it. The patient successfully completed the naming tasks but was repeatedly unsuccessful in the auditory comprehension tasks, which manifested as word-finding difficulty. Therefore, we left this cortex intact. Although the surgical window was quite narrow, the posterior insular cortices were successfully exposed. The border between the lesion and the insular white matter was obvious (Fig. 3H). A part of the inferior trunks of the middle cerebral artery, which spanned
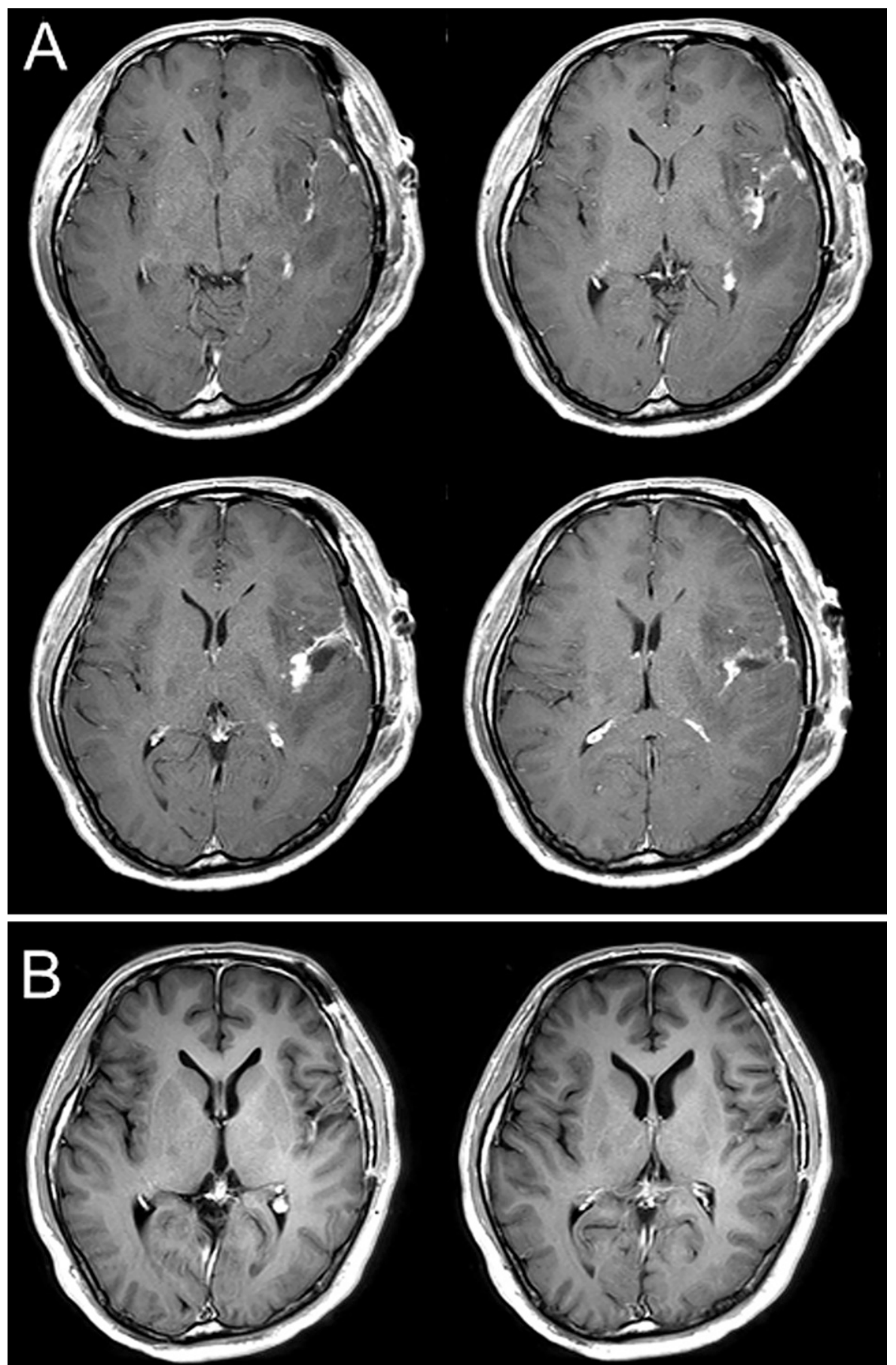

FIG. 4. A: Contrast-enhanced magnetic resonance images of the first postoperative day. B: Contrastenhanced magnetic resonance images after antituberculosis drug administration was completed. The contrast-enhancing lesions disappeared. 
the surface of the posterior insular cortices, looked whitish and was almost a part of the lesion (Fig. 3l). The intraoperative histological diagnosis indicated that the tissue was not a tumor and seemed to be neurosarcoidosis. After several submissions of the samples for intraoperative histological diagnoses, the possibility of a brain tumor, such as a glioma, was ruled out; hence further resection was discontinued. The results of the intraoperative brain mapping are summarized in Fig. 3.

The postoperative MRI images demonstrated that the contrastenhancing lesions were mostly removed (Fig. 4A). The patient did not exhibit any apparent neurological deficits, and his pure tone audiometry was confirmed to be normal after the surgery. As suggested by the neurophysiological tests, the scores associated with auditory detection were lower than the preoperative scores, including those for digit span, auditory detection task, memory updating test, and paced auditory serial addition test in the clinical assessment for attention (Table 1). He was reported to frequently ask for questions to be repeated during the rehabilitation. These findings suggested that he did not have problems in his pure tone audiometry but had problems in speech audiometry after the partial resection of his left Heschl's gyrus. The histological diagnosis was tuberculoma although his chest computed tomography images did not indicate any lesions in the lungs. After antituberculosis drug administration, he was discharged from the hospital. The patient has been observed in the outpatient department since then, and the contrast-enhancing lesions have almost disappeared (Fig. 4B). Although the scores associated with auditory detection decreased 2 weeks after the surgery, they improved 4 months after the surgery (Table 1). The patient and his family granted permission to publish this case report.

\section{Discussion}

\section{Observations}

In this article, we have reported a safe approach to the dominant posterior insular lesion by using a part of the left Heschl's gyrus as a surgical corridor after brain mapping. To the best of our knowledge, this transsylvian and trans-Heschl's gyrus approach is the first to approach a posterior insular lesion. Although the surgical approach to the insular lesions is challenging, the effectiveness of awake craniotomy in minimizing the postoperative neurological deficits, especially for cases in a dominant hemisphere, has been established. ${ }^{8}$ Regarding surgical procedures in dominant hemispheric cases, most studies have reported that the surgeries were performed based on the brain mapping results. ${ }^{1-4}$ The main differences lies in whether the transcortical or the transsylvian approach to the insular lesion is used. Although these two approaches have been compared in a cadaveric study, ${ }^{9}$ reports discussing how the posterior insula in a dominant hemisphere should be approached when the cortical mapping indicates that the perisylvian cortices have verbal functions at the level of the posterior insular lesions are quite limited. This lacuna could presumably be because the transsylvian approach is also quite difficult at this level. Duffau emphasized that splitting the sylvian fissure at this level is almost impossible because the posterior insula is located deeper than the anterior insula, and bifurcated or bridging superficial sylvian veins are frequently seen at the posterior ramus of the sylvian fissure. ${ }^{5}$ Therefore, in this case, Heschl's gyrus was considered to be a suitable surgical corridor based on the preoperative MRI fiber-tracking images even if the sylvian fissure was not fully opened.

Heschl's gyrus is the longest and the most anterior gyrus in the transverse temporal gyri. In this case, because the cortex located anterior to the area, which was used as a surgical corridor, possessed verbal functions, it was considered to be a part of the STG (area * in Fig. 3). Thus, the cortex used as a surgical corridor was judged to be Heschl's gyrus (area ** in Fig. 3). The cortex located posterior to Heschl's gyrus and had verbal functions was considered to be the transverse temporal gyrus (area *** in Fig. 3). Therefore, in this case, Heschl's gyrus was selectively removed to serve as a surgical corridor. The functions of the left Heschl's gyrus were comprehensively analyzed through intraoperative brain mapping and pre- and postoperative neurophysiological tests. Accordingly, Heschl's gyrus did not have verbal functions, whereas the transverse temporal gyrus located posterior to Heschl's gyrus did have verbal functions. The pre- and postoperative neurophysiological tests indicated that the resection of the left Heschl's gyrus seemed to be associated with the temporary impairment of speech audiometry. However, no problems were identified during the pure tone audiometry performed immediately after the surgery. These results suggest that partial resection of the left Heschl's gyrus is associated with temporary speech auditory impairment.

Heschl's gyrus is known to be the primary auditory area, and its auditory functions have been previously examined in radiological studies and surgical cases. Bilateral Heschl's gyri were activated in response to the stimulation of sound in a functional MRI study that included healthy volunteers. ${ }^{10}$ Based on two resected cases of tumors in the left Heschl's gyrus, a previous report suggested that lesions in this gyrus can be resected without verbal or auditory mapping during the surgery. ${ }^{6}$ In this case, the resection of the left Heschl's gyrus did not result in permanent verbal or auditory impairment; thus, our findings support this suggestion. However, the verbal functions of Heschl's gyrus have not been fully understood. According to morphological studies, the size of the planum temporale and Heschl's gyrus in the left hemisphere are larger than that in the right hemisphere. ${ }^{11,12} \mathrm{~A}$ previous study has reported that the structural asymmetry of Heschl's gyrus between the left and right hemispheres appears to be associated with verbal functions. ${ }^{13}$ Recently, Heschl's gyrus-associated region in which five fiber tracts intersect has been reported to be a key region functioning as a hub for language and auditory functions. ${ }^{6}$ In this case, the patient manifested temporary impairment of speech recognition, which was probably because partial resection of the left Heschl's gyrus caused a dysfunction of this network between the verbal and auditory fibers in this region. On the other hand, this patient did not exhibit any problems in pure tone audiometry, probably because sound detection is regulated by bilateral Heschl's gyri. In the present case, the findings of intraoperative brain mapping and neurophysiological tests suggested that the left Heschl's gyrus might be involved in converting the detected sound into language comprehension.

There are some limitations in the surgical approach applied in this case. The transsylvian and trans-Heschl's gyrus approach was useful and wide enough for resecting the left posterior insular lesion in the present case. However, it remains unclear whether this approach is feasible for larger lesions, especially in cases where the lesions extend posteriorly. In this case, the transverse temporal gyrus that was posteriorly adjacent to Heschl's gyrus had verbal functions, whereas Heschl's gyrus did not. Thus, performing corticectomy for the transverse temporal gyrus to posteriorly create wider surgical windows might be impossible based on this result. Moreover, it is still uncertain whether Heschl's gyrus can be safely resected under an awake condition. Further investigations are 
required to conclude the feasibility and usefulness of this approach for dominant posterior insular lesions.

\section{Lessons}

In this case, we safely approached the posterior insular lesions in a dominant hemisphere using the left Heschl's gyrus as a surgical corridor. The patient manifested temporary impairment of speech recognition after the surgery according to the intraoperative verbal mapping and neurophysiological tests but did not exhibit any permanent neurological deficits. Hence, the transsylvian and trans-Heschl's gyrus approach, along with intraoperative brain mapping, can be a viable option for resecting dominant posterior insular lesions.

\section{References}

1. Duffau H, Moritz-Gasser S, Gatignol P. Functional outcome after language mapping for insular World Health Organization Grade II gliomas in the dominant hemisphere: experience with 24 patients. Neurosurg Focus. 2009;27(2):E7.

2. Rey-Dios R, Cohen-Gadol AA. Technical nuances for surgery of insular gliomas: lessons learned. Neurosurg Focus. 2013;34(2):E6.

3. Sanai N, Polley MY, Berger MS. Insular glioma resection: assessment of patient morbidity, survival, and tumor progression. J Neurosurg. 2010;112(1):1-9.

4. Vigo V, Zanabria Ortiz R, Paganelli SL, da Costa MDS, Campos Filho JM, Chaddad-Neto F. Awake craniotomy for removal of left insular cavernous malformation. World Neurosurg. 2019;122:209.

5. Duffau H. Surgery of Insular Gliomas. Prog Neurol Surg. 2018; 30:173-185.

6. Silbergeld DL. Tumors of Heschl's gyrus: report of two cases. Neurosurgery. 1997;40(2):389-392.

7. Fernández L, Velásquez C, García Porrero JA, de Lucas EM, Martino J. Heschl's gyrus fiber intersection area: a new insight on the connectivity of the auditory-language hub. Neurosurg Focus. 2020;48(2):E7.

8. Alimohamadi M, Shirani M, Shariat Moharari R, et al. Application of awake craniotomy and intraoperative brain mapping for surgical resection of insular gliomas of the dominant hemisphere. World Neurosurg. 2016;92:151-158.

9. Benet A, Hervey-Jumper SL, Sánchez JJ, Lawton MT, Berger MS. Surgical assessment of the insula. Part 1: surgical anatomy and morphometric analysis of the transsylvian and transcortical approaches to the insula. J Neurosurg. 2016;124(2): 469-481.

10. Javad F, Warren JD, Micallef $C$, et al. Auditory tracts identified with combined fMRI and diffusion tractography. Neuroimage. 2014;84: 562-574.

11. Geschwind N, Levitsky W. Human brain: left-right asymmetries in temporal speech region. Science. 1968;161(3837):186-187.

12. Penhune VB, Zatorre RJ, MacDonald JD, Evans AC. Interhemispheric anatomical differences in human primary auditory cortex: probabilistic mapping and volume measurement from magnetic resonance scans. Cereb Cortex. 1996;6(5):661-672.

13. Dorsaint-Pierre R, Penhune VB, Watkins KE, et al. Asymmetries of the planum temporale and Heschl's gyrus: relationship to language lateralization. Brain. 2006;129(Pt 5):1164-1176.

\section{Disclosures}

Dr. Hirose reported grants from Eisai and Chugai outside the submitted work. No other disclosures were reported.

\section{Author Contributions}

Conception and design: Nakae, Ohba. Acquisition of data: Nakae, Kumon, Kojima, Higashiguchi, Sato, Inamoto. Analysis and interpretation of data: Nakae, Kojima, Higashiguchi, Sato, Inamoto, Mukaino, Hirose. Drafting the article: Nakae. Critically revising the article: Ohba, Hirose. Reviewed submitted version of manuscript: Ohba, Inamoto, Hirose. Administrative/technical/material support: Kuriyama, Mukaino, Hirose. Study supervision: Ohba, Hirose.

\section{Correspondence}

Shunsuke Nakae: Fujita Health University, Aichi, Japan. snakae.1977@ gmail.com. 